\title{
La mejora de la regulación en España como política pública: análisis y evolución
}

\author{
Better Regulation in Spain as a public policy: \\ analysis and development
}

\author{
Marisa Álvarez Suárez* \\ Ministerio de Economía, Industria y Competitividad \\ marialuisa.alvarez@mineco.es
}

\section{RESUMEN}

La «mejora de la regulación» ha de entenderse como una política pública de corte transversal y de carácter indispensable que debe funcionar como premisa de desarrollo de cualquier intervención pública para garantizar la consecución de sus principales objetivos al mínimo coste.

En España han sido importantes los esfuerzos realizados en las últimas décadas para implantar una cultura de mejora regulatoria y para recoger en el ordenamiento jurídico los principales instrumentos y principios de aplicación de la misma. No obstante, aún existen retos pendientes que podrán ser únicamente abordados eficientemente si se mantiene un elevado compromiso político al respecto.

En este artículo, se analizan las diferentes fases de implantación de la mejora de la regulación en España recogiendo sus principales hitos regulatorios y el posible margen de profundización aún existente.

\section{PALABRAS CLAVE}

Regulación eficiente, instrumentos y principios de mejora regulatoria.

\begin{abstract}
«Better regulation» can be considered as an indispensable horizontal public policy that must action as an implementation's premise of any public intervention in order to ensure that its main goals are achieved at minimum costs.

Spain has made important efforts in recent decades to develop a culture of regulatory reform and to establish the main tools and principles of better regulation in the regulatory framework. However, there are remaining challenges that can be addressed effectively only if it remains a high political commitment.

In this paper, we review the different Better regulation periods in Spain by stressing the main regulatory milestones and the possible room for improvement.
\end{abstract}

\section{KEYWORDS}

Smart regulation, Better regulation principles and tools.

* Técnico Comercial y Economista del Estado. Las opiniones vertidas en este artículo son responsabilidad exclusiva de la autora y no se corresponden necesariamente con los de ninguna institución. 


\section{SUMARIO}

1. Introducción. 2. Metodología. 3. Análisis descriptivo. Evolución de la Implantación en España de la Mejora de la Regulación. 3.1. Los inicios (1999-2008). Impulso de las primeras iniciativas. 3.2. El desarrollo (20092011). La expansión a todos los ámbitos administrativos. 3.3. La consolidación (2012-2015). 4. Discusión: El margen de profundización y los retos pendientes. 5. Conclusión. 6. Referencias bibliográficas y documentales.

\section{INTRODUCCIÓN}

El término «mejora de la regulación» procede de los anglicismos «Better Regulation», «Regulatory Reform» o «Smart Regulation», conceptos de compleja traducción y concreción que se han ido definiendo y desarrollando en el ámbito de la Unión Europea (UE) y de la Organización para la Cooperación y el Desarrollo Económico (OCDE) en las últimas tres décadas².

La mejora de la regulación, buena regulación, regulación eficiente o regulación inteligente podría definirse como un conjunto de herramientas y principios de actuación en la intervención pública para garantizar que se cumplen los objetivos previstos al mínimo coste (eliminando los posibles «fallos de estado» al reducir al mínimo el posible impacto negativo de la intervención pública).

La mejora de la regulación es pues una amalgama de procedimientos y sistemas de funcionamiento que deben aplicarse durante todo el ciclo de intervención de una política pública en las diferentes fases ex ante, de ejecución y expost (planeamiento, elaboración, aprobación, implementación y seguimiento, revisión y evaluación) para garantizar que ésta se realiza de la mejor forma posible. Funciona como una premisa de base a aplicar antes, durante y después del desarrollo de cualquier política pública independientemente de su naturaleza. Pero también debe considerarse que la asunción e implementación de los principios e instrumentos base de la mejora regulatoria por parte de las Administraciones públicas se configura en esencia como una política pública en sí misma. En consecuencia, es posible analizar y estudiar su evolución y características definitorias así como el posible margen de profundización existente al respecto.

\section{METODOLOGÍA}

El objetivo fundamental de este artículo es realizar un estudio o análisis descriptivo de la evolución de la implementación de la política de la mejora de la regulación en España en las últimas décadas con el objetivo de, a partir del análisis del contexto actual en relación a las mejores prácticas internacionales recomendadas y realizadas al respecto, proponer posibles vías de profundización y mejora.

La metodología empleada en la elaboración de este artículo se ha basado fundamentalmente en el análisis de fuentes primarias y secundarias. Así, para la realización del análisis descriptivo:

- Se ha realizado una recopilación y revisión de diversa normativa de rango legal y reglamentario y otras actuaciones -programas, planes de actuación, convenios, acuerdos, etc.- de la Administración General del Estado (AGE) de España en el periodo comprendido entre 1990 y la actualidad.

- Se han analizado diversas memorias, informes y otras fuentes documentales de diferentes instituciones u organismos de carácter nacional (Consejo de Unidad de Mercado, Comisión de Reforma de las Administraciones Públicas, Consejo de Transparencia, etc.) con competencias relacionadas con la mejora regulatoria.

Para la realización del análisis en relación a las posibles vías de mejora y profundización del estado de situación actual:

- Se ha realizado una revisión de referencias y documentos de trabajo relativos al ámbito objeto de este artículo a nivel internacional, básicamente de la UE y de la OCDE así como de diferentes países pioneros en la materia especialmente el Reino Unido.

2 Han sido muchas y variadas las actuaciones y las publicaciones en el ámbito de la UE y de la OCDE en relación a la mejora regulatoria. Por citar algunas de las más recientes por parte de la Comisión de la UE cabría señalar la publicación del documento de trabajo Better Regulation Guidelines y el Better regulation Tookbox en 2015 y en el ámbito de la OCDE la publicación de los documentos Promoting Inclusive Growth through Better Regulation: The Role of Regulatory Impact Assessment, en febrero de 2016, el OECD Regulatory Policy Outlook en 2015 y el informe emitido en 2014 Spain: From Administrative Reform to Continuous Improvement. 
- Se ha procedido al estudio de diversos artículos y referencias documentales publicados por diversos expertos en el tema.

\section{ANÁLISIS DESCRIPTIVO. EVOLUCIÓN DE LA IMPLANTACIÓN EN ESPAÑA DE LA MEJORA DE LA REGULACIÓN}

Los primeros antecedentes en España a nivel de la Administración General del Estado de lo que podríamos identificar como instrumentos parciales o principios programáticos de mejora regulatoria -antes incluso de que se empezara a hablar de ésta como política pública- se recogieron en la década de los 90 a través básicamente de las siguientes normas.

- La Ley 30/1992, de 26 de noviembre, de Régimen Jurídico de las Administraciones Públicas y del procedimiento administrativo común en su artículo 3 que recogía como principios generales de actuación de las Administraciones públicas los de eficacia, jerarquía, descentralización, desconcentración y coordinación.

- La Ley 6/1997, de 14 de abril, de Organización y Funcionamiento de la Administración General del Estado cuyos artículos 3 y 4 hacían referencia a los principios de organización y funcionamiento y de servicio al ciudadano.

- La Ley 50/1997, de 27 de noviembre, del Gobierno que en su artículo 24 ya recogía en el procedimiento de elaboración de los reglamentos la obligatoriedad de sometimiento a audiencia pública y de acompañamiento de memoria e informes preceptivos.

No obstante los primeros impulsos decididos traducidos en iniciativas concretas no se empezaron a implementar hasta llegado el año 2000 (ver en Cuadro 1 año de implantación en España de los instrumentos de mejora regulatoria que pueden ser desarrollados en las diferentes fases del ciclo de vida de una política pública). Desde entonces hasta el momento actual es posible identificar tres etapas diferenciadas caracterizadas por diferentes hitos de implantación de la mejora regulatoria.

CUADRO 1. INSTRUMENTOS DE MEJORA REGULATORIA Y AÑo DE IMPLANTACIÓN

\begin{tabular}{|c|c|c|c|}
\hline $\begin{array}{l}\text { POSIBLES INSTRUMENTOS MEJORA REGULATORIA } \\
\text { (año de implantación en España) }\end{array}$ & $\begin{array}{l}\text { Ex } \\
\text { ante }\end{array}$ & $\begin{array}{l}\text { En la } \\
\text { ejecución }\end{array}$ & $\begin{array}{c}\text { Ex } \\
\text { post }\end{array}$ \\
\hline $\begin{array}{l}\text { Plan o programas anuales } \\
2005 \text { (PNR)-2016 (Plan normativo) }\end{array}$ & $x$ & $x$ & $x$ \\
\hline $\begin{array}{l}\text { Transparencia informes } \\
2013\end{array}$ & $x$ & & \\
\hline $\begin{array}{l}\text { Consulta y participación previa } \\
2016\end{array}$ & $x$ & & \\
\hline $\begin{array}{l}\text { One in one out } \\
2013\end{array}$ & $x$ & $x$ & \\
\hline $\begin{array}{l}\text { Planes reducción cargas (convenios y acuerdos) } \\
1999\end{array}$ & $x$ & & $x$ \\
\hline $\begin{array}{l}\text { Mecanismos de disciplina } \\
2013 \text { (LGUM y Consejo Transparencia) }\end{array}$ & & $x$ & $x$ \\
\hline $\begin{array}{l}\text { Memorias de impacto } \\
2009\end{array}$ & $x$ & & \\
\hline $\begin{array}{l}\text { Evaluación externa } \\
2006 \text { (AEVAL) }\end{array}$ & & & $x$ \\
\hline $\begin{array}{l}\text { Evaluación y seguimiento interno } \\
2016\end{array}$ & & $x$ & $x$ \\
\hline $\begin{array}{l}\text { Transparencia y comunicación de resultados } \\
2013\end{array}$ & & $x$ & $x$ \\
\hline
\end{tabular}




\begin{tabular}{|c|c|c|c|}
\hline $\begin{array}{l}\text { POSIBLES INSTRUMENTOS MEJORA REGULATORIA } \\
\text { (año de implantación en España) }\end{array}$ & $\begin{array}{c}\text { Ex } \\
\text { ante }\end{array}$ & $\begin{array}{c}\text { En la } \\
\text { ejecución }\end{array}$ & $\begin{array}{c}\text { Ex } \\
\text { post }\end{array}$ \\
\hline $\begin{array}{l}\text { Entradas en vigor fijas } \\
2016\end{array}$ & & $x$ & \\
\hline $\begin{array}{l}\text { Organismo independiente de evaluación ex ante } \\
2016\end{array}$ & $x$ & & \\
\hline Labores de difusión de cultura regulatoria & $\mathrm{X}$ & $\mathrm{X}$ & $\mathrm{X}$ \\
\hline $\begin{array}{l}\text { Procesos de peer review } \\
2015 \text { (Doing Business Subnacional) }\end{array}$ & $x$ & $x$ & $x$ \\
\hline $\begin{array}{l}\text { Administración electrónica } \\
2007-2016\end{array}$ & $\mathrm{X}$ & $x$ & $x$ \\
\hline $\begin{array}{l}\text { Inclusión de principios en Ley } \\
2009\end{array}$ & $x$ & $x$ & $x$ \\
\hline $\begin{array}{l}\text { Screening de regulación existente } \\
2007 \text { (Directiva Servicios), } 2012 \text { (LGUM) }\end{array}$ & & $x$ & $x$ \\
\hline
\end{tabular}

Fuente: Elaboración propia.

\subsection{Los inicios (1999-2008). Impulso de las primeras iniciativas}

Entre 1999 y 2009 al impulso de las mejores prácticas europeas e internacionales al respecto empiezan a introducirse y a desarrollarse los primeros instrumentos de regulación eficiente que se institucionalizan a través de distintos medios:

- Programas Nacionales de Reformas -PNR- en el marco de la Agenda de Lisboa ${ }^{3}$ (a partir de 2005) en los cuales se planifican los objetivos de política económica incluyendo las iniciativas de mejora regulatoria.

- Sucesivos Planes de Simplificación Administrativa (desde 1999) y Acuerdos de Consejo de Ministros para la reducción de cargas administrativas.

- Firma de Convenios de colaboración con otras Administraciones públicas (AA.PP) y con organismos representativos de los operadores económicos para la identificación y reducción de cargas administrativas.

- Aprobación por Acuerdo de Consejo de Ministros del 22 de julio de 2005 de las Directrices de técnica normativa destinadas a mejorar la calidad de la regulación.

- Aprobación del Real Decreto 951/2005, de 29 de julio, por el que se establece el marco general para la mejora de la calidad en la Administración General del Estado.

- Creación de la Agencia Estatal de Evaluación de las Políticas Públicas y la Calidad de los Servicios (AEVAL) mediante el Real Decreto 1418/2006, de 1 de diciembre, por el que se aprueba el Estatuto de la Agencia Estatal de Evaluación de las Políticas Públicas y la Calidad de los Servicios aprobado por la habilitación dada al Gobierno en el artículo 3 de la Ley 28/2006, de 18 de julio, de Agencias Estatales para la mejora de los servicios públicos.

- Aprobación de la Ley 11/2007, de 22 de junio, de acceso electrónico de los ciudadanos a los Servicios Públicos.

En definitiva, esta primera etapa se caracterizaría por lo siguiente:

- La buena regulación se focaliza en dos ámbitos: por una parte la reducción de cargas administrativas -con el establecimiento de objetivos específicos a medio y largo plazo- a través de la mejora de la cooperación entre AA.PP y la colaboración con representantes de la sociedad civil (Confede-

${ }^{3}$ La Agenda de Lisboa era un plan de la Unión Europea aprobado en el año 2000 cuyo objetivo era convertir la economía de la UE en la más competitiva del mundo para el año 2010, basándose en una estrategia de tipo global basada en el conocimiento, el empleo y la cohesión social. 
ración Española de Organizaciones Empresariales, Cámaras de Comercio, etc.) y por otra parte el fomento de la calidad de los servicios prestados por la Administración.

- Como especial hito de este periodo en relación a los instrumentos específicos de mejora regulatoria cabría destacar la creación de la AEVAL como instrumento para la evaluación expost externa de las políticas públicas. Su creación vino precedida de un informe específico elaborado por una Comisión de Expertos de reconocido prestigio en diferentes ámbitos que, con el objetivo de realizar una propuesta de puesta en marcha del organismo, analizó concienzudamente entre otras cuestiones la experiencia internacional al respecto, y la situación existente en España.

- No existe un plan general -más allá del PNR- de mejora regulatoria ni instrumentos normativos de rango legal que regulen con carácter general la obligatoriedad de las AA.PP de atender a determinados principios (exceptuando, como se ha comentado anteriormente los específicos de carácter programático que ya estaban recogidos en la Ley 30/1992, de 26 de noviembre y en la Ley 6/1997, de 14 de abril).

- La comprensión y asimilación de la cultura de buena regulación en toda su extensión se circunscribe a la órbita del entonces Ministerio de Economía y Hacienda, del Ministerio de Administraciones públicas y posteriormente de Política Territorial y del Ministerio de Presidencia que son los organismos competentes de las actuaciones implementadas durante este periodo y las que realizan un seguimiento al respecto de la iniciativa normativa de la AGE en el marco de la Comisión Delegada del Gobierno de Asuntos Económicos (CDGAE) y de la Comisión General de Secretarios de Estado y Subsecretarios. No obstante empieza a existir una vocación expansiva a todos los ámbitos administrativos. Así, esta asimilación de la cultura de mejora regulatoria empieza en este periodo a consolidarse a través de los trabajos preparatorios necesarios para la transposición de la Directiva 2006/123/CE del Parlamento Europeo y del Consejo, de 12 de diciembre de 2006, relativa a los servicios en el mercado interior -Directiva de servicios- ${ }^{4}$.

- Cuestión aparte de especial relevancia, sería la aprobación en este periodo de la Ley 15/2007, de 3 de julio, de Defensa de la Competencia lo que supone el desarrollo de la política de promoción a la competencia (advocacy) como instrumento especial de fomento de la regulación eficiente para eliminar barreras y obstáculos innecesarios y desproporcionados a la actividad económica y en consecuencia promover la competencia efectiva en los mercados.

\subsection{El desarrollo (2009-2011). La expansión a todos los ámbitos administrativos}

El periodo entre 2009 y 2011 se caracteriza, además de por la continuación de uso de los instrumentos previstos en la anterior etapa, por la aprobación de diferentes proyectos normativos en los que se recoge de manera expresa los principales instrumentos y principios de mejora regulatoria y la obligación de su respeto y fomento por parte de las Administraciones públicas.

En concreto se desarrollan las siguientes iniciativas:

- La aprobación del Real Decreto 1083/2009, de 3 de julio, por el que se regula la memoria del análisis de impacto normativo y del Acuerdo de Consejo de Ministros por el que se aprueba la Guía Metodológica de elaboración del análisis de impacto normativo que incluye el método simplificado de medición de cargas administrativas basado en el modelo de costes estándar. Tal y como consideraba el preámbulo de la norma el objetivo de este Real Decreto era precisar el contenido de las memorias, estudios e informes sobre la necesidad y oportunidad de las normas proyectadas, así como de la memoria económica y del informe sobre el impacto por razón de género, que deben acompañar a los anteproyectos de ley y a los proyectos de reglamento, a los que hacen referencia los artículos 22 y 24 de la Ley 50/1997, de 27 de noviembre, del Gobierno. De esta forma, a través de este Real Decreto y de la Guía Metodológica se sentaron las bases para Administración General del Estado del «Impact Assesment», instrumento principal de mejora regulatoria por el

4 La Directiva de servicios planteaba un periodo de transposición de cuatro años. En marzo de 2007 se creó por Acuerdo de la Comisión Delegada del Gobierno de Asuntos Económicos un grupo interministerial encargado de la preparación de los trabajos para la transposición de dicha Directiva. Los trabajos (también con Comunidades Autónomas y Entidades Locales) que finalmente desembocaron en la aprobación de las Leyes de transposición -véase siguiente apartado- contribuyeron en este periodo a la extensión de la nueva cultura regulatoria y la asunción de ésta como política pública a impulsar y aplicar por las todas las Administraciones. 
cual se evalúa con carácter ex ante el impacto de la misma sobre sus destinatarios y sobre la ciudadanía en general.

- La aprobación de la Ley $17 / 2009$, de 23 de noviembre, sobre el libre acceso a las actividades de servicios y su ejercicio (denominada Ley Paraguas). Esta ley transpone al ordenamiento jurídico español con carácter horizontal la Directiva de servicios. La norma recoge por primera vez una serie obligaciones vinculadas con el cumplimiento de principios de mejora regulatoria (en relación a la transparencia, la simplificación de procedimientos, la aplicación del principio de necesidad y proporcionalidad, de eficacia nacional y de no discriminación, etc.). No obstante su ámbito de aplicación se circunscribe al de los servicios dentro del ámbito de aplicación de la Directiva que incluye notables excepciones como los transportes, los servicios sanitarios, las comunicaciones electrónicas, los servicios audiovisuales, el juego, etc. Asimismo esta Ley creó el Comité para la Mejora de la Regulación de las Actividades de Servicios (CMR).

- La aprobación de la Ley 25/2009, de 22 de diciembre, de modificación de diversas leyes para su adaptación a la Ley sobre el libre acceso a las actividades de servicios y su ejercicio (denominada Ley Ómnibus). La Ley Ómnibus supuso a la adaptación de diversas normas sectoriales de rango legal a los principios establecidos en la Ley paraguas incluso en algunos sectores que hubieran estado fuera de su ámbito de aplicación como por ejemplo transportes, postal o comunicaciones electrónicas. Asimismo de forma especialmente ambiciosa, supuso la extensión de la aplicación de los principios de mejora regulatoria a toda intervención pública a través de la modificación de la Ley 30/1992, de 26 de noviembre, de Régimen Jurídico de las Administraciones Públicas y del Procedimiento Administrativo Común mediante la adición de un nuevo artículo 39 bis ${ }^{5}$.

- La aprobación de la Ley 2/2011, de 4 de marzo, de Economía Sostenible supuso la sistematización a través del Capítulo I de su Título primero de los principios e instrumentos de mejora de la calidad de la regulación. En concreto el artículo 4 reguló la obligación para el conjunto de las Administraciones públicas de aplicar los principios de mejora regulatoria en el ejercicio de su iniciativa normativa y el artículo 5 la obligación de impulsar tres instrumentos concretos (la consulta pública y la evaluación ex ante y ex post). Esta Ley además, entre otras iniciativas, supuso la extensión de la aplicación de los principios de mejora regulatoria al ámbito local través de la modificación del artículo 84 bis ${ }^{6}$ de la Ley $7 / 1985$, de 2 de abril, Reguladora de las Bases del Régimen Local y modificó el estatus del CMR para ampliar su ámbito de actuación al de todas las actividades económicas.

En definitiva, este periodo de desarrollo se caracteriza por:

- Un impulso decidido de la aplicación de los principios e instrumentos de mejora de la regulación de forma que se extiende definitivamente tanto de iure como de facto como una política pública a aplicar y respetar por todos los ámbitos de la administración.

- Una cierta focalización, gracias al impulso de la Directiva de servicios, de las políticas de mejora regulatoria en relación a su impacto sobre las actividades económicas, es decir de aprobación de iniciativas vinculadas a la reducción de obstáculos y barreras para las operadores económicos. En este sentido, cabe recordar que mejorar la regulación no implica necesariamente desregular ni incrementar la regulación. No obstante, es cierto que la existencia de lo que se ha venido a denomi-

\footnotetext{
${ }^{5}$ Artículo 39 bis. Principios de intervención de las Administraciones Públicas para el desarrollo de una actividad (hoy derogado):

1. Las Administraciones Públicas que en el ejercicio de sus respectivas competencias establezcan medidas que limiten el ejercicio de derechos individuales o colectivos o exijan el cumplimiento de requisitos para el desarrollo de una actividad, deberán elegir la medida menos restrictiva, motivar su necesidad para la protección del interés público así como justificar su adecuación para lograr los fines que se persiguen, sin que en ningún caso se produzcan diferencias de trato discriminatorias.

2. Las Administraciones Públicas velarán por el cumplimiento de los requisitos aplicables según la legislación correspondiente, para lo cual podrán comprobar, verificar, investigar e inspeccionar los hechos, actos, elementos, actividades, estimaciones y demás circunstancias que se produzcan.

${ }^{6}$ Artículo 84 bis. (hoy modificado):

(...) con carácter general, el ejercicio de actividades no se someterá a la obtención de licencia u otro medio de control preventivo. No obstante, podrán someterse a licencia o control preventivo aquellas actividades que afecten a la protección del medio ambiente o del patrimonio histórico-artístico, la seguridad o la salud públicas, o que impliquen el uso privativo y ocupación de los bienes de dominio público, siempre que la decisión de sometimiento esté justificada y resulte proporcionada. En caso de existencia de licencias o autorizaciones concurrentes entre una entidad local y alguna otra Administración, la entidad local deberá motivar expresamente en la justificación de la necesidad de la autorización o licencia el interés general concreto que se pretende proteger y que éste no se encuentra ya cubierto mediante otra autorización ya existente.
} 
nar red tape (regulación excesiva o redundante) hace que la implementación de políticas de mejora regulatoria de tipo económico se hayan frecuentemente traducido en una eliminación de obstáculos o barreras al acceso y al ejercicio de actividades económicas y, por tanto, hayan venido asociadas a políticas de liberalización económica.

\subsection{La consolidación (2012-2015)}

En 2012 la implementación de toda política pública se vio condicionada por el contexto de acentuación de crisis económica. Así, en 2012 el Producto Interior Bruto (PIB) de la economía española retrocedió un 2,9\% (datos actualizados del Instituto Nacional de Estadística -INE- en 2016), alcanzando la tasa de paro un $26,02 \%$ en el cuarto trimestre. La prima española de riesgo alcanzó en julio de ese año su máximo histórico (649 puntos básicos en su cotización intradía). La contracción económica española era muy superior a la del conjunto de la UE, lo que reflejaba la existencia de deficiencias estructurales diferenciales en la economía española y la necesidad de acometer reformas de carácter estructural para aumentar el potencial de crecimiento del PIB, la creación de empleo y la reducción de los desequilibrios.

La estrategia económica implementada en los siguientes años con el objetivo de revertir esta situación -recogida en los sucesivos PNR- se basó en cuatro pilares fundamentales; la consolidación fiscal para garantizar la sostenibilidad de las finanzas públicas; la reforma estructural de los mercados de factores productivos (mediante la reforma laboral y la reforma financiera y bancaria); la reforma de los mercados de bienes y servicios (centrada en diversas iniciativas generales y sectoriales basadas en la aplicación de la mejora regulatoria en todas las actividades económicas) y, estrechamente vinculada a esta cuestión, la reforma de la Administración. Así, en el ámbito de la mejora de la regulación se pueden señalar las siguientes iniciativas:

\section{- La creación de la Comisión de Reforma de las Administraciones Públicas ${ }^{7}$ y de la Oficina para la Ejecución de la Reforma de la Administración}

Por Acuerdo de Consejo de Ministros en octubre de 2012 se crea la Comisión de Reforma de las Administraciones públicas (CORA) a la que se le encomienda la realización de un análisis general del funcionamiento de las Administraciones Públicas en España con el objetivo de identificar las áreas de mejora y realizar propuestas al respecto. Para la realización de este análisis se utilizaron herramientas informáticas que involucraron a una multitud de empleados públicos en todos los ámbitos administrativos (designándose responsables de los Ministerios que coordinan las actuaciones y responsables de ejecución de cada una de las medidas). El estudio fue realizado a través de cuatro Subcomisiones: Duplicidades administrativas, Simplificación administrativa ${ }^{8}$, Gestión de Servicios y Medios Comunes y Administración Institucional. El Informe final ejecutivo de la CORA fue presentado al Consejo de Ministros en junio de 2013. Este exhaustivo informe identificaba lo que consideraba ineficiencias y redundancias competenciales a corregir recogiendo un total de 217 propuestas de medidas.

El Real Decreto 479/2013, de 21 de junio, creó la Oficina para la Ejecución de la Reforma de la Administración (OPERA) con rango de Subsecretaría, con el objetivo de supervisar, impulsar y coordinar la ejecución de las dichas medidas (desde 2014 asume también funciones relacionadas con la transparencia). Este Real Decreto preveía la realización de informes trimestrales y anuales de seguimiento a presentar al Consejo de Ministros. Según el último informe de seguimiento presentado, hasta mayo de 2016 el número de medidas implantadas era de 193 (grado de ejecución superior al 85\%).

\section{- El Programa de Garantía de la Unidad de Mercado}

La CDGAE en mayo de 2012 creó el Grupo de trabajo interministerial para la garantía de la unidad de mercado, y en julio de ese mismo año aprobó un Programa de Trabajo de Garantía de Unidad de Mercado -PGUM- cuyo objetivo principal era analizar las medidas para eliminar los obstáculos y las barreras a la

7 Como iniciativa especialmente relevante en el ámbito de la reforma de las Administraciones Públicas cabría señalar la aprobación de la Ley $27 / 2013$, de 27 de diciembre, de racionalización y sostenibilidad de la Administración Local.

${ }^{8}$ Esta Subcomisión de simplificación administrativa elaboró el Manual de Simplificación Administrativa y de Reducción de Cargas para la Administración General del Estado cuya publicación se acordó por Acuerdo de Consejo de Ministros el 19 de Septiembre de 2014. 
actividad económica producto de la fragmentación del mercado nacional y establecer un modelo regulatorio de las actividades económicas más eficiente.

EI PGUM se componía de varias fases entre las que destacaba la aprobación de un marco normativo de referencia - La Ley 20/2013, 9 de diciembre, de garantía de la unidad de mercado (la LGUM)- y la puesta en marcha del Plan de Racionalización Normativa -para lo que se usó una plataforma informática específica denominada "gum»- con el objetivo de adaptar la normativa sectorial existente a los principios establecidos en dicha Ley (Plan en el que estuvieron involucrados más de 160 personas sólo a nivel de la AGE).

La LGUM ahonda en los principios de mejora regulatoria establecidos en la Ley paraguas y los hace extensivos a todas las actividades económicas. Pero además establece una novedad: para garantizar el cumplimiento de estos principios y evitar la inacción de las Administraciones públicas en la adaptación sectorial de los mismos la LGUM establece procedimientos privilegiados - de gestión mediante la Secretaría del Consejo para la Unidad de Mercado y la Comisión Nacional de los Mercados y la Competencia- mediante los cuales los operadores pueden interponer reclamaciones e informar sobre obstáculos y barreras a la unidad de mercado. Hasta enero de 2017 la Secretaría del Consejo para la Unidad de Mercado había recibido más de 335 reclamaciones y había hecho público en su página web más de 200 informes de quejas recibidas. Por su parte, la CNMC había interpuesto 21 recursos contenciosos administrativos ante la Audiencia Nacional por actos y disposiciones generales de las Administraciones públicas que consideraba contrarios a los principios recogidos en la LGUM.

\section{- La aprobación de la Ley 14/2013, de 27 de septiembre, de apoyo a los emprendedores y su internacionalización}

Esta Ley recoge una serie de medidas orientadas a apoyar al emprendedor y la actividad empresarial y a favorecer el denominado "clima de negocios». En especial cabría hacer referencia como especiales instrumentos de mejora regulatoria a lo recogido en sus artículos 36, 37 y 38 relativos a la revisión del clima de negocios a través de las mejoras regulatorias, a la denominada regla one in one out (sólo se puede crear una carga administrativa nueva si se elimina otra ya existente) y a la reducción de cargas estadísticas para las empresas. En relación a las medidas de revisión de clima de negocios a través de la elaboración de indicadores y el intercambio de buenas prácticas cabría señalar como medidas que desarrollan lo establecido en esta Ley la elaboración del Doing Business en España - subnaciona/ ${ }^{9}$ publicado en septiembre de 2015 realizado por el Banco Mundial y la introducción de un nuevo módulo sobre entorno empresarial y buena regulación en la Encuesta de Indicadores de Confianza Empresarial del Instituto Nacional de Estadística. Asimismo en enero de 2015 se aprobó un Acuerdo de Consejo de Ministros que establecía medidas para desarrollar el comentado principio one in one out de forma que la compensación de cargas se establece preferiblemente en el ámbito de cada departamento ministerial.

\section{- La aprobación de la Ley 19/2013, de 9 de diciembre, de transparencia, acceso a la información pública y buen gobierno}

La Ley 19/2013, de 9 de diciembre, tiene por objeto «ampliar y reforzar la transparencia de la actividad pública, regular y garantizar el derecho de acceso a la información relativa a aquella actividad y establecer las obligaciones de buen gobierno que deben cumplir los responsables públicos así como las consecuencias derivadas de su incumplimiento». Esta Ley refunde y desarrolla principios de buena regulación en materia de transparencia y buen gobierno que ya se encontraban presentes, aunque de forma parcial, en la normativa española. Como cuestión de especial relevancia prevé la puesta en disposición de los ciudadanos de la información de relevancia jurídica que se ha utilizado en el proceso de elaboración de iniciativas normativas. Asimismo, al igual que la LGUM, en su ámbito de actuación esta Ley incluye una novedad destinada a servir de cierre de la Ley y de mecanismo de disciplina de las Administraciones públicas: crea un procedimiento privilegiado a través de una reclamación potestativa ad hoc sustitutiva de los recursos administrativos ordinarios de la que conocerá el Consejo de Transparencia y Buen Gobierno, organismo de naturaleza independiente de nueva creación en la propia Ley. A 31 de marzo de 2017 el Consejo había recibido un total de

9 El Doing Business en España 2015 realiza un análisis comparado de las regulaciones existentes en las diecisiete Comunidades Autónomas, en Ceuta y Melilla y en cinco puertos de referencia. Se analizan las regulaciones que inciden sobre el ciclo de vida de una PYME: apertura de una empresa, obtención de permisos de construcción, obtención de electricidad, registro de propiedades y comercio transfronterizo. 
2.853 iniciativas ciudadanas y hasta septiembre de 2016 se habían realizado 1.140 resoluciones, tanto de la Administración General del Estado como de las Comunidades Autónomas con las que el Consejo ha firmado convenio (Principado de Asturias, Cantabria, La Rioja, Castilla-La Mancha, Extremadura, Ceuta y Melilla).

\section{- Reforma integral del procedimiento administrativo común y el régimen jurídico del sector público}

El periodo de consolidación de la política de mejora regulatoria en España se vio culminado en este periodo con la reforma integral ${ }^{10}$ que supuso la aprobación en paralelo de dos normas: La Ley 39/2015, de 1 de octubre, del Procedimiento Administrativo Común de las Administraciones Públicas y La Ley 40/2015, de 1 de octubre, de Régimen Jurídico del Sector Público. Ambas normas recogen en relación a la mejora regulatoria, entre otras muchas reformas, varias de las recomendaciones realizadas por el informe de 2014 de la OCDE "Spain: From Administrative Reform to Continous Improvement".

Estas dos normas suponen una reforma profunda en la organización y ordenación de las Administraciones tanto en su funcionamiento interno y en sus relaciones entre si (la Ley 40/2015, de 1 de octubre) como en su funcionamiento externo es decir en sus relaciones con la ciudadanía (la Ley 39/2015, de 1 de octubre). Las iniciativas concretas que contienen se basan en los principios de mejora regulatoria en la medida en que están orientadas al incremento de la transparencia, de la seguridad jurídica, de la racionalización administrativa y de la mejora de la eficiencia de la Administración y de la calidad normativa. Para ello ambas leyes prevén el impulso y la modernización de varios de los clásicos instrumentos de mejora regulatoria. Se prevé por ejemplo:

- La sistematización de los principios de mejora regulatoria.

- El impulso de la administración electrónica ad intra y ad extra.

- La elaboración de un Plan Anual Normativo coordinado por el Ministerio de Presidencia.

- La obligatoriedad de realización de una consulta pública a través de la página web del departamento competente con anterioridad a la redacción de las propuestas normativas.

- El reforzamiento del contenido de la Memoria del Análisis de Impacto Normativo con la inclusión de los impactos sobre la competitividad y la unidad de mercado y el denominado test pyme (evaluación del impacto de la normativa para la pequeñas y medianas empresas).

- La atribución de funciones específicas relacionadas con la coordinación y calidad normativa al Ministerio de la Presidencia. En concreto se le atribuye la revisión de «El cumplimiento o congruencia de la iniciativa con los proyectos de reducción de cargas administrativas o buena regulación que se hayan aprobado en disposiciones o acuerdos de carácter general para la Administración General del Estado».

- El refuerzo de la evaluación ex post de las iniciativas normativas que han sido adoptadas a través de un Informe Anual de Evaluación a aprobar por el Consejo de Ministros.

- La fijación de fechas de entrada en vigor de aquellas normas con impacto en las actividades económicas y profesionales de carácter fijo ( 1 de julio y 2 de enero).

La entrada en vigor de ambas normas se produjo en octubre del año 2016 y algunas de las medidas que contienen requieren de un desarrollo reglamentario aún pendiente ${ }^{11}$ por lo que aún es demasiado pronto para valorar el impacto final de estas iniciativas.

En definitiva, puede afirmarse que este último periodo de 2012 a 2015 ha servido para consolidar la senda emprendida en la etapa anterior en relación a la mejora de la regulación. Como cuestiones claves de este periodo podría entonces señalarse:

10 Entre otras muchas derogaciones y modificaciones esta reforma ha supuesto la derogación de la Ley $30 / 1992$, de 26 de noviembre, de la Ley 11/2007, de 22 de junio, de la Ley 6/1997, de 14 de abril y de la Ley 28/2006, de 18 de julio (por detallar algunas de las anteriormente citadas en este artículo) así como la derogación de varios artículos de la Ley 2/2011, de 4 de marzo y la modificación de la Ley 50/1997, de 27 de noviembre.

11 En el ámbito de la Administración General del Estado varias de las cuestiones comentadas ya han sido recientemente objeto de desarrollo. Véase, por ejemplo, la Orden PRE/1590/2016, de 3 de octubre, por la que se publica el Acuerdo del Consejo de Ministros de 30 de septiembre de 2016, por el que se dictan instrucciones para habilitar la participación pública en el proceso de elaboración normativa a través de los portales web de los departamentos ministeriales o el Real Decreto 286/2017, de 24 de marzo, por el que se regulan el Plan Anual Normativo y el Informe Anual de Evaluación Normativa de la Administración General del Estado y se crea la Junta de Planificación y Evaluación Normativa. 
- La puesta en marcha de programas (PGUM o CORA) de amplio espectro en relación a su ámbito de aplicación que han involucrado a una multitud de empleados públicos lo que ha servido para finalmente conformar la mejora de la regulación como política pública en si misma a respetar por todos los poderes públicos.

- La implementación de mecanismos adhoc de cierre y disciplina (mecanismos en el marco de la LGUM y de la Ley 19/2013, de 9 de diciembre) orientados a dar voz a la ciudadanía y a las empresas para convertirles en participes de las reformas.

- La aprobación de una reforma integral en el marco del procedimiento administrativo y el régimen jurídico del sector público que supone la implementación para España de las buenas prácticas más avanzadas a nivel internacional de mejora regulatoria en estos ámbitos.

\section{DISCUSIÓN: EL MARGEN DE PROFUNDIZACIÓN Y LOS RETOS PENDIENTES}

España ha partido de un relativo retraso en relación a otras economías avanzadas respecto a la asunción de la mejora de la regulación como política pública por las diferentes Administraciones. No obstante, en las últimas dos décadas, como se puede observar a través del análisis de las múltiples iniciativas realizadas, se han dado pasos muy importantes para reducir y cerrar esta brecha diferencial.

Debe señalarse especialmente el sustancial impacto económico de las medidas relacionadas con la mejora de la regulación -en especial de aquellas relacionadas con la regulación eficiente de las actividades económicas- en términos de crecimiento y empleo. Desde un punto de vista económico el beneficio de la regulación eficiente es evidente. Crea condiciones más favorables para la inversión, al incrementar la seguridad jurídica y la reducción de cargas administrativas, fomenta la competencia y promueve el crecimiento económico. Así, por ejemplo, el Programa Nacional de Reformas para 2016 recogía, entre otras, un impacto estimado relevante en términos de crecimiento del PIB y del empleo de iniciativas como la reforma de la administración, la aplicación de la LGUM y la Ley de emprendedores en un horizonte temporal a largo plazo (de 10 años desde la aprobación de la medida concreta).

CUADRO 2. IMPACTO ECONÓMICO DE REFORMAS DE MEJORA DE LA REGULACIÓN

\begin{tabular}{lcc}
\hline MEDIDA & $\begin{array}{c}\text { Efecto PIB } \\
\text { (largo plazo) }\end{array}$ & $\begin{array}{c}\text { Efecto Empleo } \\
\text { (largo plazo) }\end{array}$ \\
\hline Reforma de la Administración: Local y CORA & 1,41 & 0,41 \\
\hline Ley de garantía de la unidad de mercado & 1,6 & 0,86 \\
\hline Ley de apoyo a emprendedores (incentivos no fiscales) & 0,31 & 0,15 \\
\hline
\end{tabular}

Fuente: Programa Nacional de Reformas (2016)

Las reformas orientadas a la mejora de la regulación tienen, por tanto, un importante papel para la generación de un crecimiento económico sólido y estable, capaz de generar empleo. A este impacto económico se suman además los beneficios relacionados con la mejora de la prestación de servicios a la ciudadanía -y consecuentemente sobre el bienestar- con carácter general. Pero para ello, es fundamental que las reformas emprendidas desplieguen todos sus efectos y potencial.

En este sentido, cabría por tanto considerar el margen existente aún de mejora y los posibles retos pendientes tanto normativos como institucionales. Tanto la Comisión de la UE como la OCDE resaltan con carácter general cuestiones como la necesidad de que exista un compromiso político en el tiempo, una política de formación continua a los empleados públicos y de comunicación externa, una involucración de todas las Administraciones afectadas y que se pueda identificar claramente a los artífices de la política pública (en la Comisión de la UE por ejemplo el Vicepresidente primero es, entre otras cuestiones, Comisario de Better Regulation). Específicamente el informe de la OCDE de 2010 sobre mejora de la regulación en España reconocía el avance decidido producido desde el año 2000 en la aprobación de iniciativas concretas en este ámbito y los esfuerzos técnicos y políticos realizados. No obstante, se señalaban diferentes deficiencias en el enfoque adoptado (falta de estrategia integradora, necesidad de mayor cooperación entre Administraciones y mayor impulso político, mejor estrategia de comunicación, necesidad de mayor extensión de cultura 
de mejora regulatoria, etc.) y propuestas de mejora al respecto. En relación a estas, y otras cuestiones, y, a pesar, de los avances producidos en los últimos años, en España:

- Sigue existiendo una cierta dispersión, desde el punto de vista normativo. Es decir, a pesar de las diferentes modificaciones normativas e iniciativas de refundición realizadas, siguen existiendo diferentes disposiciones en las que se recogen los instrumentos y los principios de mejora regulatoria con definición no del todo coincidente -véase por ejemplo el Anexo I-.

- Existe igualmente una dispersión desde el punto de vista de «cabezas visibles» encargados de liderar esta política pública. Así, atendiendo por ejemplo a la distribución de competencias según sus propios Reales Decretos de estructura en la legislatura 2012-2016, la mejora de la regulación desde el punto de vista económico parecía estar más liderada desde el extinto Ministerio de Economía y Competitividad o incluso desde el entonces Ministerio de Industria, Energía y Turismo (en relación al clima empresarial para las pequeñas y medianas empresas). Sin embargo, materias como la administración electrónica o la reducción de cargas administrativas con carácter general se encontraban atribuidas el extinto Ministerio de Hacienda y Administraciones públicas ${ }^{12}$. La reestructuración ministerial tras las elecciones de octubre de 2016 supuso un avance en la medida en que las funciones relacionadas con el clima empresarial quedaron integradas en el nuevo Ministerio de Economía, Industria y Competitividad. No obstante, tanto dicho Ministerio, como el ahora Ministerio de Hacienda y Función Pública y el nuevo Ministerio de Presidencia y para las Administraciones Territoriales siguen asumiendo diferentes funciones en relación a la mejora de la regulación de las actividades económicas y cuestiones como la administración electrónica, la calidad normativa o la reducción de cargas administrativas.

- En relación estrecha con lo anterior, puede considerarse que aún existe margen para una mayor refundición de los diferentes instrumentos y un enfoque más integrador y sistematizado que señalice específicamente la mejora de la regulación como política pública en sí misma. Por ejemplo, en el Reino Unido -país pionero y considerado líder en la materia- un Better Regulation Executive establece el marco de referencia o la agenda de mejora regulatoria con carácter anual que todos los departamentos gubernamentales deben respetar y existe un Comité independiente (el Regulatory Policy Comittee) encargado de la supervisión de las evaluaciones de impacto de los proyectos normativos. Asimismo, en el Reino Unido existe un marco general de revisión del stock de normativa existente iniciado en 2011 conocido como el Red Tape Challenge. Similares enfoques sistematizados, aunque focalizados en determinadas áreas según las particularidades concretas de cada país, se han seguido en países como Holanda, Bélgica, Estados Unidos o Suecia.

- Los programas de formación sistemáticos y continuos de los empleados públicos se han planteado únicamente en áreas específicas (por ejemplo en relación a la aplicación de la Ley 19/2013, de 9 de diciembre ${ }^{13}$, o respecto a la reforma del procedimiento administrativo) por lo que aún existe margen para una mayor profundización en este aspecto. Es esencial que la «cultura» de la buena regulación sea conocida y compartida por los empleados públicos y políticos encargados de su implementación para garantizar su efectiva asunción como política pública.

- Igualmente los programas de comunicación externa y de difusión de los diferentes derechos y mecanismos a disposición de la ciudadanía para impulsar la mejora regulatoria se han realizado en áreas concretas y de forma parcial.

- En relación a la regulación eficiente de las actividades económicas (la aplicación de los principios de buena regulación a la remoción de obstáculos al acceso y al ejercicio de actividades económicas) a pesar de las iniciativas implementadas -en especial la aprobación de la LGUM- aún es posible igualmente considerar vías para una mayor profundización ${ }^{14}$. Así, por ejemplo la propia OCDE a

12 Por otra parte, las cuestiones vinculadas a la calidad normativa de los proyectos se han realizado históricamente también por parte de las Secretarías Generales Técnicas de los departamentos ministeriales aunque el Ministerio de Presidencia ha asumido igualmente nuevas funciones al respecto en la Ley 40/2015, de 1 de octubre.

13 En relación a la transparencia y buen gobierno la propia Disposición adicional séptima de La Ley 19/2013, de 9 de diciembre, establecía que «El Gobierno aprobará un plan formativo en el ámbito de la transparencia, dirigido a los funcionarios y personal de la Administración General del Estado, acompañado a su vez de una campaña informativa dirigida a los ciudadanos».

${ }_{14} \mathrm{El}$ posible margen de mejora de nuestro país en relación a la regulación de tipo económico es visible a través del análisis de los más representativos indicadores internacionales de competitividad y de clima de negocios como por ejemplo el Global Competitiveness Index del Foro Económico Mundial de Davos, el Global Competitiveness Yearbook de la Escuela IMD en Suiza o el más conocido Doing Business del Banco Mundial. 
través de sus indicadores de mejora regulatoria -PMR- ha identificado históricamente margen de mejora en sectores concretos como el comercio minorista, el transporte y determinadas profesiones reguladas. Asimismo, organismos internacionales como el Fondo Monetario Internacional en sus Consultas del Artículo IV o el Consejo de la UE en el marco del Semestre Europeo han recomendado a España una aplicación ambiciosa de la LGUM y la realización de actuaciones concretas en áreas como el comercio minorista o los servicios profesionales.

Por otra parte, debe tenerse en cuenta que varios de los instrumentos que han sido recientemente recogidos en el ordenamiento jurídico aún están pendiente de desarrollo y de asimilación por las Administraciones públicas responsables de su aplicación. Por ejemplo la regla one in one out, la evaluación y el seguimiento interno, la aprobación del Programa anual, los nuevos impactos a incluir en las memorias de análisis de impacto de los proyectos normativos, la evaluación a realizar por el Ministerio de Presidencia, la administración totalmente electrónica etc. Para su efectiva implementación será necesaria probablemente la aprobación de desarrollos reglamentarios al respecto o de guías metodológicas, instrucciones o criterios de actuación concretos ${ }^{15}$. Sin obviar la importancia de que los principios y los instrumentos estén debidamente recogidos en el marco normativo de referencia debe garantizarse que se ponen en marcha los mecanismos prácticos para garantizar su verdadera ejecución. Así, de las actuaciones futuras que se emprendan en estos ámbitos dependerá el verdadero éxito de la implementación de estos instrumentos.

Por último, cabe señalar que las iniciativas recogidas en este artículo hacen referencia únicamente a las realizadas en el ámbito de la Administración General del Estado. Si bien todas las Comunidades Autónomas en el ámbito de sus competencias han igualmente asumido esta política pública en mayor o menor medida aprobando iniciativas normativas al respecto y participando en aquellos procesos de carácter interadministrativo (como el realizado durante la transposición de la Directiva de servicios o en los mecanismos previstos en el Programa de Garantía de la Unidad de Mercado). En el ámbito local, sin embargo (sin perjuicio de las medidas adoptadas en el ámbito de la Ley $27 / 2013$, de 27 de diciembre, de racionalización y sostenibilidad de la Administración Local) es donde es posible identificar las mayores diferencias entre corporaciones en relación a la asunción de esta política pública y, en consecuencia, donde existe un mayor margen de maniobra y profundización.

\section{CONCLUSIÓN}

La mejora de la regulación como política pública a aplicar en todas las intervenciones públicas es considerada a día de hoy una herramienta indispensable a desarrollar e implementar por todas las Administraciones.

En España han sido múltiples las iniciativas que al respecto se han llevado a cabo desde los años 90 con el objetivo de consolidar la regulación eficiente como una política pública en si misma a respetar por todos los poderes públicos. Este especial esfuerzo ha sido reconocido por diversas instituciones internacionales con competencias específicas en la materia. A día de hoy, puede decirse que todos los instrumentos de mejora de la regulación recomendados a nivel internacional en los manuales de referencia (en los diferentes documentos de la OCDE y en el Better regulation toolbox de la Comisión Europea) han sido teóricamente recogidos en nuestro marco jurídico. Es más puede incluso considerarse que España ha sido una pionera en la implantación de determinados instrumentos puestos a disposición de la ciudadanía y de los operadores económicos que funcionan como mecanismos de cierre de los principios de mejora regulatoria de forma que se evita su carácter meramente programático y se obliga a las Administraciones públicas a su efectivo cumplimiento.

No obstante aún hay margen para la profundización desde un punto de vista tanto institucional como normativo de forma que la implementación práctica de dicha política pueda desplegar todo su potencial en términos económicos y de servicio a la ciudadanía. Pero ello sólo será posible si se mantiene o incluso se refuerza el compromiso político que al respecto ha existido en las últimas décadas y se dota a esta política pública de los recursos materiales, normativos y humanos necesarios para su efectivo desarrollo técnico.

15 Para ello puede tenerse igualmente en cuenta la experiencia acumulada de los países pioneros en la materia. Por ejemplo, en el Reino Unido existe ya una implantación efectiva de una regla one in three out y un desarrollo importante de cuestiones como la evaluación de impacto económico de las medidas sobre las pequeñas y medianas empresas o las evaluaciones ex post. 


\section{REFERENCIAS BIBLIOGRÁFICAS Y DOCUMENTALES}

BALSA PASCUAL, C. (2006), "La Better Regulation", Papeles de Evaluación, núm. 1, Madrid. En línea: http://www. aeval.es/comun/pdf/papeles_evaluacion/Papeles_de_Evaluacion_nx1.pdf (consulta el 3 de noviembre de 2016).

BALSA PASCUAL, C. y GUTIÉRREZ CARRIZO, I. (2008), “La mejora de la regulación ¿cuál es el papel de la CNC?”, Anuario de la competencia, núm. 1, Madrid.

CONSEJO PARA LA UNIDAD DE MERCADO (2015, 2017), I y II Informe del Consejo para la Unidad de Mercado. En línea: http://www.mineco.gob.es/portal/site/mineco/gum (consulta el 27de mayo de 2017).

CORA (2013), Informe de Reforma de las Administraciones públicas, Madrid. En línea: http://transparencia.gob.es/ transparencia/dam/jcr:b1c69477-9882-41a5-9f6d-5cbb46fa12b4/reforma-AAPP.PDF (consulta el 3 de noviembre de 2016).

COMISIÓN EUROPEA (2015), Commission Staff Working Document - Better Regulation Guidelines y Better Regutation "Tookbox", Bruselas. En linea: http://ec.europa.eu/smart-regulation/guidelines/docs/swd_br_ guidelines_en.pdf (consulta el 3 de noviembre de 2016).

- http://ec.europa.eu/smart-regulation/guidelines/docs/br_toolbox_en.pdf (Consulta el 3 de noviembre de 2016).

FERNÁNDEZ DÍEZ PICAZO, M. y MATEO FEITO, M. (2011), "Evaluación de Impacto Normativo en España", Presupuesto y Gasto Público 68/2012, Instituto de Estudios Fiscales, Madrid. En línea: http://www.ief.es/ documentos/recursos/publicaciones/revistas/presu_gasto_publico/68_06.pdf (consulta el 3 de noviembre de 2016).

KOSKE, I. et al. (2015), "The 2013 update of the OECD's database on product market regulation: Policy insights for OECD and non-OECD countries", OECD Economics Department Working Papers, núm. 1.200, OECD, Paris. En línea: $h$ ttp://dx.doi.org/10.1787/5js3f5d3n2vl-en (consulta el 25 de febrero de 2017).

MINISTERIO DE ECONOMÍA, INDUSTRIA Y COMPETITIVIDAD, Programas Nacionales de Reformas, Madrid. En

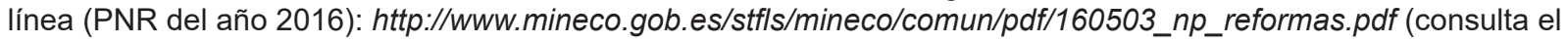
20 de febrero de 2017).

MINISTERIO DE ECONOMÍA Y HACIENDA (2010), Informe sobre la transposición de la Directiva de Servicios, Madrid. En línea: $h t t p: / / w w w . m i n e c o . g o b . e s / s t f l s / m i n e c o / e c o n o m i a / e n / a c e s / d e s t a c a d o s / D o c 3 D S . p d f$ (consulta el 3 de noviembre de 2016).

OCDE (2010), Mejora de la Regulación en Europa (España), Paris. En línea: http://www.oecdbookshop.org/get-it.php? $R E F=5 K 9 H 5 W V 4 X 5 R 1 \& T Y P E=b r o w s e$ (consulta el 3 de noviembre de 2016).

OCDE (2014), Spain: From Administrative Reform to Continuous Improvement, Paris. DOI:10.1787/9789264210592en. En línea: http://www.keepeek.com/Digital-Asset-Management/oecd/governance/spain-from-administrativereform-to-continuous-improvement_9789264210592-en\#.WBs4BJ2DNjo (consulta el 3 de noviembre de 2016).

OCDE (2015), OECD Regulatory Policy Outlook, Paris. DOI:10.1787/9789264238770-en. En línea: http://www. keepeek.com/Digital-Asset-Management/oecd/governance/oecd-regulatory-policy-outlook-2015_9789264238770en\#.WBs4cJ2DNjo (consulta el 3 de noviembre de 2016).

MATEO FEITO, M. (2011), "Hacia una regulación económica más inteligente", Boletín de Información Comercial Española, núm. 3.020, Madrid. En línea: http://www.revistasice.com/CachePDF/BICE_3020_3-14_. D0E6A9A8FED5029DF819BCAFF52AAE74.pdf (consulta el 3 de noviembre de 2016).

OPERA (2016), Informe trimestral de seguimiento a Consejo de Ministros, marzo 2016, Madrid. En linea: http://www. seap.minhap.es/dms/es/web/areas/reforma_aapp/actuaciones-informes/proceso/CORA-Informe-trimestral-deseguimiento-Marzo-2016.pdf (consulta el 3 de noviembre de 2016).

\section{ANEXO I. DEFINICIÓN PRINCIPIOS MEJORA DE LA REGULACIÓN}

\begin{tabular}{ll}
\hline PRINCIPIOS & DEFINICIÓN* \\
\hline Necesidad y eficacia & $\begin{array}{l}\text { La iniciativa normativa debe estar justificada por una razón de interés general, basarse } \\
\text { en una identificación clara de los fines perseguidos y ser el instrumento más adecuado } \\
\text { para garantizar su consecución. }\end{array}$ \\
\hline Proporcionalidad & $\begin{array}{l}\text { La iniciativa que se proponga deberá contener la regulación imprescindible para atender } \\
\text { la necesidad a cubrir con la norma, tras constatar que no existen otras medidas menos } \\
\text { restrictivas de derechos, o que impongan menos obligaciones a los destinatarios. }\end{array}$ \\
\hline Seguridad Jurídica & $\begin{array}{l}\text { La iniciativa normativa se ejercerá de manera coherente con el resto del ordenamiento } \\
\text { jurídico, para generar un marco normativo estable, predecible, integrado, claro y de cer- } \\
\text { tidumbre, que facilite su conocimiento y comprensión y, en consecuencia, la actuación y } \\
\text { toma de decisiones de las personas y empresas. }\end{array}$ \\
\hline
\end{tabular}


Transparencia

Sostenibilidad financiera

Estabilidad presupuestaria

Simplicidad de cargas

No discriminación
Se publicará de forma periódica y actualizada la información cuyo conocimiento sea relevante para garantizar la transparencia de su actividad relacionada con el funcionamiento y control de la actuación pública.

Las Administraciones Públicas posibilitarán el acceso sencillo, universal y actualizado a la normativa en vigor y los documentos propios de su proceso de elaboración, definirán claramente los objetivos de las iniciativas normativas y su justificación en el preámbulo o exposición de motivos; y posibilitarán que los potenciales destinatarios tengan una participación activa en la elaboración de las normas.

La iniciativa normativa debe evitar cargas administrativas innecesarias o accesorias y racionalizar, en su aplicación, la gestión de los recursos públicos.

Se entenderá por sostenibilidad financiera la capacidad para financiar compromisos de gasto presentes y futuros dentro de los límites establecidos de déficit, deuda pública y morosidad de deuda comercial.

Se entenderá por estabilidad presupuestaria de las Administraciones Públicas la situación de equilibrio o superávit estructural.

La intervención de las distintas autoridades competentes garantizará que no genera un exceso de regulación o duplicidades y que la concurrencia de varias autoridades en un procedimiento no implica mayores cargas administrativas para el operador que las que se generarían con la intervención de una única autoridad.

Todos los operadores económicos tendrán los mismos derechos en todo el territorio nacional y con respecto a todas las autoridades competentes, sin discriminación alguna por razón del lugar de residencia o establecimiento.

Ninguna disposición de carácter general, actuación administrativa o norma de calidad que se refiera al acceso o al ejercicio de actividades económicas podrá contener condiciones ni requisitos que tengan como efecto directo o indirecto la discriminación por razón de establecimiento o residencia del operador económico.

Confianza mutua Las autoridades competentes, en sus relaciones, actuarán de acuerdo con el principio de confianza mutua, respetando el ejercicio legítimo por otras autoridades de sus competencias, reconociendo sus actuaciones y ponderando en el ejercicio de competencias propias la totalidad de intereses públicos implicados y el respeto a la libre circulación y establecimiento de los operadores económicos y a la libre circulación de bienes y servicios por todo el territorio nacional.

Eficacia Nacional Los actos, disposiciones y medios de intervención de las autoridades competentes relacionados con el libre acceso y ejercicio de la actividad económica tendrán eficacia en todo el territorio nacional.

Necesidad y proporcionalidad (en el acceso y ejercicio de actividades económicas)

Las autoridades competentes que en el ejercicio de sus respectivas competencias establezcan límites al acceso a una actividad económica o su ejercicio o exijan el cumplimiento de requisitos para el desarrollo de una actividad, motivarán su necesidad en la salvaguarda de alguna razón imperiosa de interés general. Cualquier límite o requisito establecido deberá ser proporcionado a la razón imperiosa de interés general invocada, y habrá de ser tal que no exista otro medio menos restrictivo o distorsionador para la actividad económica.

Principio general de intervención de las AA.PP (incluye evaluación ex ante y obligación de evaluación ex post)
Las Administraciones Públicas que, en el ejercicio de sus respectivas competencias, establezcan medidas que limiten el ejercicio de derechos individuales o colectivos o exijan el cumplimiento de requisitos para el desarrollo de una actividad, deberán aplicar el principio de proporcionalidad y elegir la medida menos restrictiva, motivar su necesidad para la protección del interés público así como justificar su adecuación para lograr los fines que se persiguen, sin que en ningún caso se produzcan diferencias de trato discriminatorias. Asimismo deberán evaluar periódicamente los efectos y resultados obtenidos.

*Fuente: La concreción de los anteriores principios es una adaptación realizada a partir de la definición establecida en las siguientes leyes:

- Ley 20/2013, de 9 de diciembre, de garantía de la unidad de mercado.

- Ley 39/2015, de 1 de octubre, del Procedimiento Administrativo Común de las Administraciones Públicas.

- Ley 40/2015, de 1 de octubre, de Régimen Jurídico del Sector Público.

- Ley 19/2013, de 9 de diciembre, de transparencia, acceso a la información pública y buen gobierno.

- Ley Orgánica 2/2012, de 27 de abril, de Estabilidad Presupuestaria y Sostenibilidad Financiera. 